\title{
Le nouvel UTC(OP) fondé sur les fontaines atomiques du LNE-SYRTE
}

\section{The new UTC(OP) based on LNE-SYRTE atomic fountains}

\author{
Michel ABGRALL, Sébastien BIZE, Baptiste CHUPIN, Jocelyne GUÉNA, Philippe LAURENT, Peter \\ ROSENBUSCH, Pierre UHRICH et Giovanni Daniele ROVERA
}

LNE-SYRTE, Observatoire de Paris, PSL Research University, CNRS, Sorbonnes Universités, UPMC Univ. Paris 06, 61 avenue de l'Observatoire, 75014 Paris, France, michel.abgrall@obspm.fr.

\section{Résumé}

Cet article présente la nouvelle méthode mise en œuvre pour générer l'échelle de temps UTC(OP), la réalisation française en temps réel du Temps Universel Coordonné (UTC). Dans une première étape achevée en octobre 2012, un nouvel algorithme fondé sur l'utilisation d'un maser à hydrogène et exploitant les données des fontaines atomiques du LNE-SYRTE, a permis d'améliorer de façon significative la stabilité de UTC(OP). UTC(OP) constitue dorénavant l'une des meilleures échelles de temps au monde. Par la suite l'instrumentation servant à générer et distribuer les signaux de UTC(OP) a été entièrement renouvelée. Certains appareils sont des instruments du commerce, mais, pour les éléments clés, des dispositifs spécifiques ont été développés. En particulier, nous avons développé un commutateur permettant de basculer la distribution des signaux de UTC(OP) entre deux échelles de temps générées à partir de deux masers à hydrogène différents et fonctionnant continûment en parallèle. Ce système garantit la continuité des signaux lorsqu'il est nécessaire de changer de maser principal. Dans la nouvelle distribution, UTC(OP) est matérialisé par trois types de signaux cohérents $(100 \mathrm{MHz}, 10 \mathrm{MHz}$ et $1 \mathrm{PPS})$. Sur les trois premières années de fonctionnement, le nouvel UTC(OP) a été maintenu à moins de 10 ns de l'UTC. La différence est même inférieure à $5 \mathrm{~ns}$ si l'on exclut une courte période autour du MJD 56650.

MOTS CLÉS : MÉTROLOGIE DU TEMPS, ALGORITHME D'ÉCHELLES DE TEMPS, UTC $(k)$, ÉTALONS PRIMAIRES DE FRÉQUENCE.

\footnotetext{
Abstract

UTC(OP), the French national realization of the international Coordinated Universal Time, was redesigned and rebuilt. The first step was the implementation in October 2012 of a new algorithm based on a H-maser and on atomic fountain data. Thanks to the new implementation, the stability of UTC $(O P)$ was dramatically improved and $U T C(O P)$ competes with the best time scales available today. The hard-
}

ware generating and distributing the UTC $(O P)$ physical signals was then replaced. Part of the new hardware is composed of commercial devices, but the key elements were specifically developed. One of them is a special switch that allows the UTC $(O P)$ signals to be derived from one of two time scales, based on two different $H$-masers, which are generated simultaneously. This insures the continuity of the UTC $(O P)$ signals even when a change of the reference H-maser is required. With the new hardware implementation, UTC $(O P)$ is made available through three coherent signals: $100 \mathrm{MHz}, 10 \mathrm{MHz}$ and $1 \mathrm{PPS}$. For more than 3 years, $U T C(O P)$ remained well below 10 ns close to UTC, with a difference even less than 5 ns if we except a short period around MJD 56650.

KEY WORDS: TIME METROLOGY, TIMESCALE ALGORITHM, UTC( $k)$, PRIMARY FREQUENCY STANDARDS.

\section{Introduction}

Par définition, on appelle « $\mathrm{UTC}(k) »$ la réalisation temps réel du Temps universel coordonné (UTC) par le laboratoire $k$. En général, la définition du temps légal d'un pays est confiée au Laboratoire national de métrologie ou à un Laboratoire désigné réalisant un $\operatorname{UTC}(k)$, comme c'est le cas en France pour le LNE-SYRTE, situé à l'Observatoire de Paris (OP). En pratique, il n'y a pas de règles strictes pour la réalisation d'un UTC $(k)$. En 1993, le Comité consultatif pour la définition de la seconde (CCDS), devenu depuis le Comité consultatif du temps et des fréquences (CCTF), a recommandé que « les écarts de temps d'un UTC $(k)$ par rapport à l'UTC restent inférieurs à $100 \mathrm{~ns}$ (à un écart type près) » [1]. En pratique, cette recommandation est suivie par plus des deux tiers des 70 laboratoires réalisant un $\operatorname{UTC}(k)$. Les capacités 
métrologiques d'un laboratoire se reflètent dans son aptitude à réaliser un $\mathrm{UTC}(k)$ qui soit stable et se maintienne proche de l'UTC. Cependant, ces qualités ne sont avérées que si l'UTC $(k)$ constitue une échelle de temps indépendante, c'est-à-dire qu'elle soit basée uniquement sur les horloges dont dispose le laboratoire, et sur les données de la Circulaire T pour le pilotage vers l'UTC.

Pendant de nombreuses années, UTC(OP) était réalisé à partir du signal fourni par une horloge à jet thermique de césium du commerce, dont la fréquence était corrigée empiriquement [2]. Une nouvelle méthode, qui repose sur le pilotage d'un maser à hydrogène au moyen d'un générateur d'écart de fréquence, a été mise en œuvre à partir du 29 octobre 2012 (MJD 56229). Le nouvel algorithme est décrit dans le paragraphe 2. Le paragraphe 3 donne une description exhaustive de la nouvelle chaîne de génération de UTC(OP). Le paragraphe 4 présente l'analyse des résultats obtenus sur les trois premières années de fonctionnement du nouveau système.

\section{L'algorithme de UTC(OP)}

La recommandation du CCDS de 1993 [1] fournit un majorant souhaitable sur les écarts de temps entre un UTC $(k)$ et l'UTC, mais ne donne pas de contrainte sur la façon de générer un $\mathrm{UTC}(k)$. Par conséquent, il y a plusieurs interprétations possibles sur les objectifs de la réalisation d'un $\operatorname{UTC}(k)$. Dans certains cas, l'algorithme mis en place privilégie la stabilité de l'intervalle de temps unitaire (c'est-à-dire la fréquence), tout en acceptant que l'échelle de temps s'éloigne de temps à autre de l'UTC [3]. Dans le cas de UTC(OP), notre objectif est de produire un signal physique, disponible en temps réel, qui réalise au mieux l'UTC, en tenant compte du fait que les données d'écart à l'UTC ne sont accessibles qu'après un délai pouvant atteindre 40 jours, correspondant à la publication mensuelle de la Circulaire T par le Bureau international des poids et des mesures (BIPM). L'algorithme que nous avons développé repose sur deux hypothèses :

- l'UTC constitue l'échelle de temps la plus stable qui nous soit accessible;

- il nous est possible de générer une échelle de temps aussi stable que celle de l'UTC.

Cependant, s'il est évident que l'écart de temps entre UTC(OP) et l'UTC dépend de la stabilité des deux échelles de temps, il ne nous est pas possible, pendant le mois en cours, de prévoir la stabilité de l'UTC. À partir de ces hypothèses, nous avons identifié deux étapes pour la génération de UTC(OP) :

- premièrement, produire une échelle de temps dont l'intervalle de temps unitaire soit le plus stable possible, sans se soucier de son exactitude;

- deuxièmement, mettre en place un asservissement de fréquence et/ou de phase pour ajuster l'intervalle de temps unitaire de UTC(OP) sur celui de l'UTC, afin de maintenir l'échelle de temps aussi proche que possible de l'UTC.

L'algorithme mis en place comprend donc deux blocs distincts conceptuellement très différents.

\subsection{Premier bloc : algorithme de stabilité}

Bien qu'un maser à hydrogène de bonne qualité, comme ceux dont nous disposons, soit relativement stable et prédictible, nous tirons parti de l'ensemble de fontaines atomiques, étalons primaires et secondaires de fréquence [4-8] développés au LNE-SYRTE, pour estimer avec exactitude la fréquence du maser à hydrogène utilisé pour réaliser UTC(OP). Le premier bloc de l'algorithme constitue une boucle ouverte du type feed-forward. Il produit la majeure partie de la correction de fréquence, de l'ordre de $10^{-13}$, appliquée au maser à hydrogène. Cette partie de l'algorithme a été développée en privilégiant la robustesse, sans rechercher à atteindre la stabilité ultime, car la continuité de fonctionnement d'un $\operatorname{UTC}(k)$ constitue l'une de ses caractéristiques les plus importantes. En considérant les stabilités typiques du maser à hydrogène et des fontaines atomiques, nous avons choisi d'effectuer une mise à jour quotidienne de la correction appliquée au générateur d'écart de fréquence alimenté par le maser. Afin de tirer parti de la stabilité long terme du maser et de disposer d'un système robuste à l'absence éventuelle de données, nous estimons la fréquence du maser à partir d'une extrapolation linéaire, à la date de mise à jour de la correction, en s'appuyant sur les vingt derniers jours de données disponibles des fontaines. Ainsi, la fréquence courante du maser peut être estimée avec une incertitude meilleure que $10^{-15}$, même en cas d'absence de données causée, par exemple, par une interruption de la fontaine, des perturbations sur la génération et la distribution des signaux ou encore un incident sur le traitement automatique des données. Une période d'évaluation plus courte améliorerait très probablement la stabilité, puisque les fluctuations de fréquence du maser seraient compensées plus finement. Mais dans ce cas, quelques jours de données manquantes auraient un impact important sur l'échelle de temps. Signalons que le résultat de ce premier bloc de pilotage est équivalent à produire une échelle de temps réalisant la seconde du Système international d'unités (SI).

De plus, cette méthode de compensation de la fréquence du maser constitue un système en boucle ouverte. De cette façon, elle ne présente pas de risque d'oscillations, comme c'est le cas pour une boucle fermée. Bien qu'une analyse théorique rigoureuse du système sorte du cadre de cet article, nous pouvons raisonnablement supposer que la stabilité de l'échelle de temps produite est une réplique de celle du maser à hydrogène pour des périodes d'observations inférieures ou égales à un jour et qu'elle converge lentement sur celle de la fontaine atomique, pour des périodes d'intégration de dix jours et plus.

Le concept sous-jacent à ce système de pilotage repose sur l'utilisation de données échantillonnées 
sur des périodes plus courtes que la période de mise à jour de la correction, extrapolées à la date courante, après avoir appliqué un filtrage des éventuelles données aberrantes. Ce concept avait été mis en pratique dans un contexte complètement différent, pour réaliser l'asservissement de fréquence d'un oscillateur à quartz sur un laser He-Ne à $633 \mathrm{~nm}$ stabilisé sur une raie d'absorption de l'iode [9].

\subsection{Second bloc : pilotage vers l'UTC}

La seconde partie de l'algorithme constitue une boucle d'asservissement permettant de piloter finement la fréquence de UTC(OP), afin de maintenir l'échelle de temps proche de l'UTC. Les corrections de fréquence associées sont typiquement de quelques $10^{-16}$. Ce pilotage doit être compatible avec le délai de mise à disposition de la Circulaire T, publiée mensuellement par le BIPM. De plus, les données portent sur les résultats du mois écoulé. Il y a donc un délai de l'ordre d'une dizaine de jours entre la date de la dernière donnée et la date courante lors de la publication de la Circulaire T. Les données se présentent sous la forme des écarts de temps [UTC-UTC $(k)]$ échantillonnés tous les cinq jours aux dates MJD finissant par 4 ou 9.

Pour réaliser ce pilotage, la méthode la plus simple serait de réaliser une boucle à verrouillage de phase (Phase Lock Loop, PLL) où l'écart de temps entre l'UTC et UTC(OP) constitue la variable d'entrée et le pilotage de fréquence appliqué au générateur d'écart de fréquence, la variable de contrôle. Cette technique produit un intégrateur, du fait de la relation entre phase et fréquence. Cependant, les règles de stabilité des systèmes d'asservissement [10] imposent que le gain du PLL doit être bien en dessous de l'unité à la fréquence inverse du délai du système (entre la date courante et la date de la dernière donnée). Nous avons donc opté pour une boucle combinant un asservissement de fréquence (Frequency Lock Loop, FLL) et un PLL.

Le FFL consiste à évaluer la différence de fréquence entre l'UTC et UTC(OP) au cours de la période couverte par la dernière Circulaire $\mathrm{T}$ parue. La valeur obtenue constitue la première partie du pilotage mensuel qui sera maintenu constant pour le mois suivant. Cela constitue une boucle d'ordre 1 présentant un gain unitaire à la période d'échantillonnage de 1 mois du système. Il n'y a donc pas de risque d'oscillation. Pour la partie PLL, l'écart de temps à compenser est estimé en extrapolant les données de la Circulaire $\mathrm{T}$ à la date courante de mise à jour du pilotage. La correction de fréquence de ce PLL correspond à l'écart de temps obtenu, divisé par $60 \mathrm{~d}$. Cette méthode, utilisant une extrapolation, nous permet en quelque sorte de résoudre le problème du délai de publication de la Circulaire T. De plus, le faible gain de 0,5 du PLL à la période d'échantillonnage de la boucle empêche toute suroscillation.

Notre objectif est d'obtenir la meilleure estimation possible de la différence de fréquence entre UTC $(\mathrm{OP})$ et l'UTC au cours de la période de la dernière Circulaire T

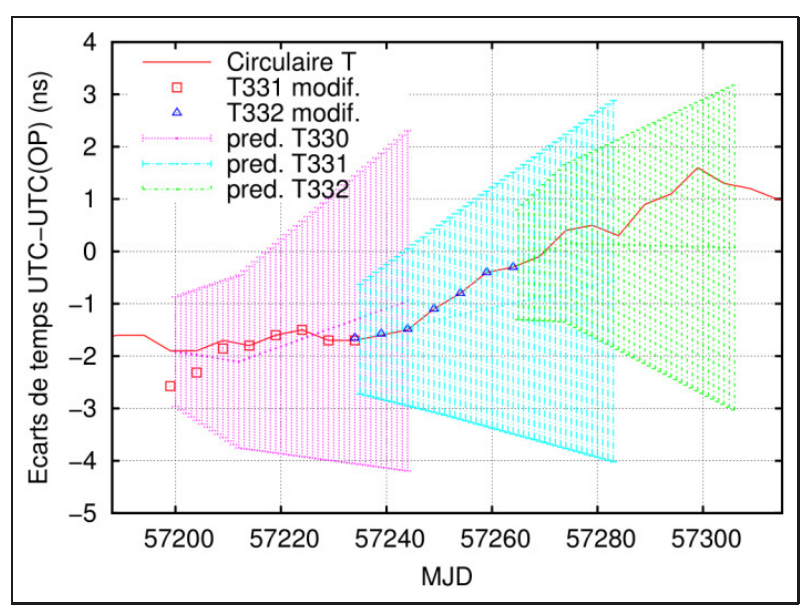

Fig. 1. - Écarts de temps $[U T C-U T C(O P)]$; données extraites de la Circulaire $\mathrm{T}$ (points donnés aux dates MJD se terminant par 4 ou 9) et prédictions de $[U T C-U T C(O P)]$ (un point par jour).

parue, ainsi que la prédiction de l'écart de temps à la date courante, en tenant compte du pilotage en cours. En particulier, il faut prendre en compte le fait que le pilotage du mois précédent était appliqué sur environ le premier tiers de la période couverte par la Circulaire T. Nous devons donc corriger la valeur des premiers points issus de la Circulaire $\mathrm{T}$ de la différence entre la correction de fréquence courante et celle du mois précédent. Cette opération est mise en évidence dans la figure 1, où les carrés rouges fournissent l'exemple du calcul effectué pour la mise à jour du pilotage, après la publication de la Circulaire T 330, au MJD 57212. Dans ce graphique, les carrés au-delà de ce MJD sont superposés à la courbe rouge, qui représente $[U T C-U T C(O P)]$ tel que publié dans la Circulaire T. Cependant, aux dates précédant le MJD 57212, il y avait une différence 6,04 × 10 entre le pilotage en cours et celui du mois précédent. Les points précédant cette date ont donc été corrigés en conséquence. La figure 1 présente également des prédictions des écarts entre l'UTC et UTC(OP), estimées au moment de la mise à jour du pilotage, en magenta, cyan et vert, respectivement pour les Circulaires T 330, 331 et 332. La rupture de pente sur ces prédictions, clairement visible pour les calculs issus des Circulaires T 330 et 332, correspondent à la date de mise à jour du pilotage. Ceci est moins visible pour la Circulaire T 331, car la valeur du pilotage obtenue était très proche de celle de la Circulaire T 330. Les barres d'erreurs présentées ont été estimées empiriquement en considérant une incertitude de 1 ns sur l'estimation de l'écart de temps et de 50 ps par jour, soit environ $5 \times 10^{-16}$, sur la prédiction de la fréquence de UTC(OP). Ces valeurs sont réalistes et peuvent être justifiées en analysant les données de la Circulaire T. En effet, les incertitudes publiées sur le lien réalisant le raccordement à l'UTC varient entre 1,0 ns et $1,5 \mathrm{~ns}$. On peut également supposer qu'un éventuel biais sur ce lien ne changerait pas de façon significative d'un mois à l'autre. D'autre part, l'incertitude de fréquence provient de la combinaison de l'incertitude sur l'estimation de la fréquence sur la période couverte par la Circulaire T 
avec la stabilité des deux échelles de temps, UTC et UTC(OP), au cours de la même période. Les résultats présentés dans le paragraphe 4 confirment une incertitude de $5 \times 10^{-16}$ sur la prédiction de fréquence sur une période d'observation de 1 mois.

L'algorithme présenté produit donc trois corrections de fréquence du maser à hydrogène pouvant facilement être appliquées via le même actuateur. En pratique, le générateur d'écart de fréquence alimenté par le maser reçoit chaque jour une correction qui est la somme du FLL et du PLL, mise à jour chaque mois et gardée constante jusqu'à la publication de la Circulaire $\mathrm{T}$ suivante, ainsi que de la correction du feed-forward, mise à jour quotidiennement.

\section{Mise en place du nouvel UTC(OP)}

L'algorithme présenté au paragraphe précédent a été mis en œuvre en octobre 2012, en utilisant le générateur d'écart de fréquence et le système de distribution qui étaient alors en fonctionnement. Les seules autres modifications effectuées ont porté sur la mise en place d'un traitement automatique de données issues des fontaines atomiques et le remplacement du signal à $5 \mathrm{MHz}$ initialement fourni par l'horloge à jet thermique de césium par celui du maser à hydrogène de référence. En parallèle, nous avons développé et testé de nouveaux instruments à bas bruit fonctionnant à $100 \mathrm{MHz}$, afin de construire une nouvelle chaîne de génération de UTC(OP). Cette nouvelle chaîne a été caractérisée en détail et sa fiabilité a été testée en opérant des échelles de temps en parallèle à la chaîne « officielle». À partir de juin 2015, la nouvelle chaîne a été déclarée opérationnelle, et les différents équipements ont été progressivement connectés au nouveau système, tout en maintenant les deux chaînes en fonctionnement parallèle. Cette transition, qui a duré jusqu'en novembre 2015, a été complètement transparente pour les différents systèmes du laboratoire raccordés à UTC(OP). Après cette date, l'ancien système a été retiré des activités opérationnelles. Il est cependant maintenu en fonctionnement pour des tests scientifiques et à titre de redondance supplémentaire.

La figure 2 présente un synoptique simplifié de la nouvelle chaîne de génération de UTC(OP). Le système exploite un ensemble de fontaines atomiques, les étalons primaires de fréquence FO1, FO2-Cs et FOM, et l'étalon secondaire FO2-Rb, développés au LNE-SYRTE [7, 8] et qui présentent une exactitude de fréquence entre $2 \times 10^{-16}$ et $6 \times 10^{-16}$. Ces fontaines utilisent le même oscillateur local, fondé sur un oscillateur cryogénique à résonateur de saphir, développé par l'UWA (University of Western Australia) [11], verrouillé en phase sur l'un des quatre masers à hydrogène du laboratoire, avec une constante de temps de l'ordre de 1000 s. Ainsi, les fontaines tirent parti du très faible bruit de phase de l'oscillateur cryogénique et atteignent la limite du bruit de projection quantique [12]. Les fontaines atomiques mesurent la fréquence du maser à hydrogène sur les périodes plus longues que la constante de temps de la boucle d'asservissement de l'oscillateur cryogénique. Cet ensemble est en fonctionnement quasi continu depuis une quinzaine d'an-

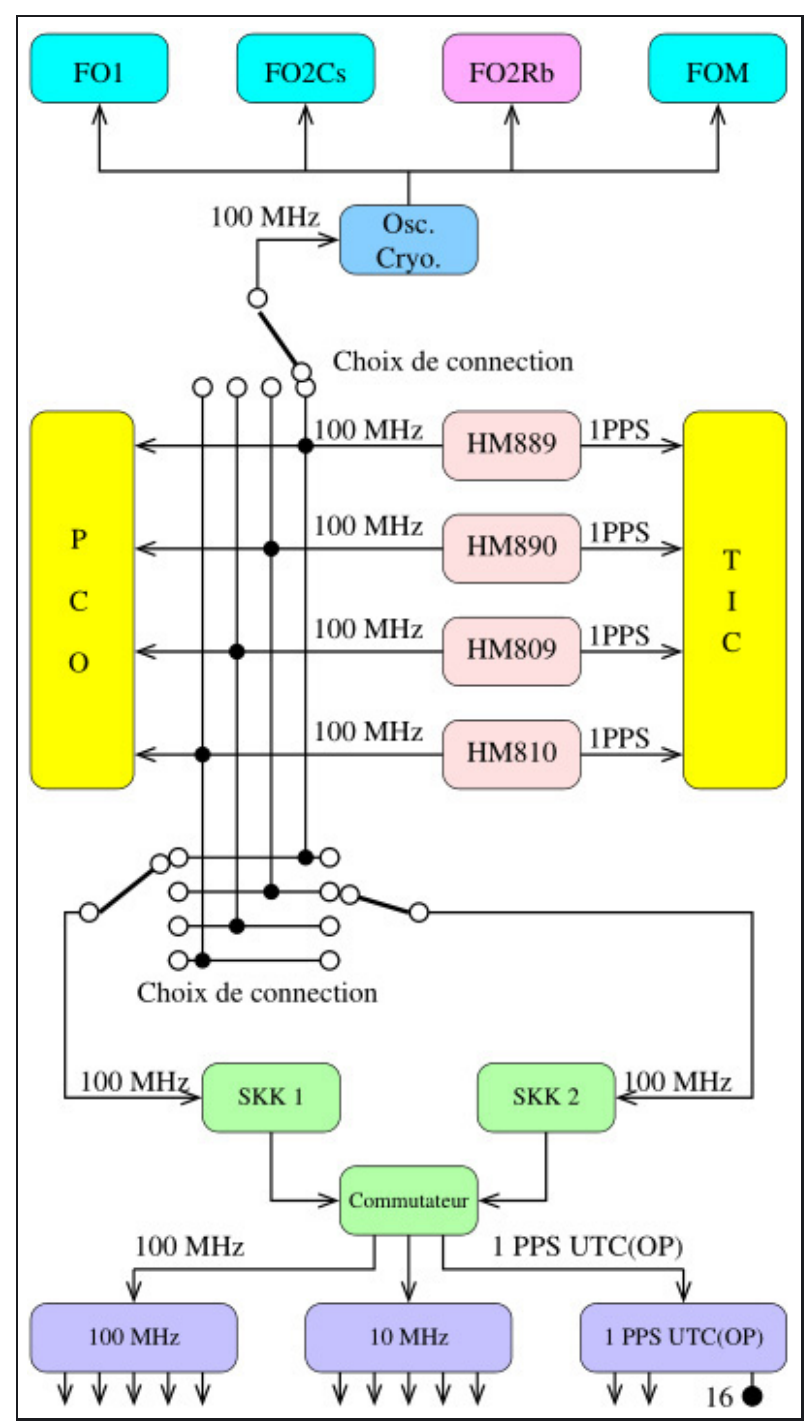

Fig. 2. - Schéma simplifié de la nouvelle chaîne de génération et de distribution de UTC(OP); PCO est un comparateur de phase fonctionnant sur des signaux à $100 \mathrm{MHz}$; TIC est un compteur d'intervalles de temps ; SKK1 et SKK2 sont des générateurs d'écart de fréquence. Le point de référence de UTC(OP) est matérialisé sur le connecteur «16» du distributeur de signaux à 1 PPS, à un seuil de déclenchement de $1 \mathrm{~V}$.

nées au laboratoire. Les références $[7,8]$ en donnent une description détaillée.

\subsection{Logiciel de réduction des données}

Nous avons développé un logiciel qui produit automatiquement toutes les heures les données cycle à cycle des fontaines, corrigées des effets systématiques. Ce logiciel calcule ensuite des données moyennes, qui sont celles effectivement utilisées dans la génération de UTC(OP), et qui servent également au suivi du fonctionnement des masers à hydrogène et des fontaines. Le format des données issues de ce traitement, stockées dans des fichiers que nous appelons des fichiers «pack», a été établi il y a plus d'une dizaine d'années pour la comparaison locale des fontaines. 
En pratique, ces valeurs moyennes sont évaluées à raison de dix points par jour sur des intervalles bien définis couvrant les époques allant de 0 à $0,1 \mathrm{~d}, 0,1 \mathrm{~d}$ à $0,2 \mathrm{~d}$, etc. Afin de prendre en compte la dérive de fréquence de l'oscillateur local, nous effectuons une régression linéaire sur les données de chaque intervalle, en utilisant les données cycle à cycle des fontaines. Le traitement inclut un filtrage itératif des points aberrants. À la fin de ce processus, si le nombre de points valides est suffisant, les données de l'intervalle sont considérées comme valides et la valeur résultante du point moyen correspond à l'interpolation des données cycle à cycle à la date milieu de l'intervalle.

Ce traitement a été développé afin de réduire la quantité de données à manipuler, en considérant le bruit des fontaines atomiques et celui des différents oscillateurs du système, dominés par du bruit blanc de fréquence. Sur cet intervalle de 0,1 jour, les valeurs moyennes sont toujours dominées par du bruit blanc. Cette durée a cependant été choisie car si nous avions opté pour une période plus longue, il aurait été plus difficile de détecter des évènements non statistiques.

Le logiciel de traitement de données exploite de plus les données de comparaison entre les quatre masers à hydrogène mesurées à l'aide d'un ensemble de comparateurs de phase de signaux à $100 \mathrm{MHz}$ (PCO) et de compteurs d'intervalles de temps sur des signaux un pulse par seconde (signaux 1 PPS), comme indiqué dans la figure 2. Ceci nous permet d'étalonner quasiment en temps réel la fréquence de chacun de nos quatre masers par rapport aux quatre fontaines atomiques. Nous avons donc à notre disposition les données nécessaires pour générer une échelle de temps à partir de chacun des masers. Il serait certes possible de combiner ces données de masers, mais nous avons cependant choisi la solution simple, et donc plus fiable, de générer UTC(OP) à partir d'un maser piloté par l'une des fontaines. Le logiciel permet, à partir d'un fichier de paramètres de choisir le maser et la fontaine utilisés. Il est à noter également que grâce à la disponibilité des données de fréquence de chaque maser, le maser servant de référence aux fontaines atomiques n'est pas forcément celui utilisé pour produire UTC(OP).

\subsection{Développement de nouveaux instruments}

Afin d'améliorer la stabilité à court terme de l'échelle de temps qui était limitée par le synthétiseur $5 \mathrm{MHz}$ d'ancienne génération, nous avons développé, en collaboration avec la société SKK Electronics [13], un nouveau type de générateur d'écart de fréquence spécialement conçu pour fonctionner à $100 \mathrm{MHz}$. Les figures 3 et 4 présentent respectivement le spectre de bruit de phase et l'écart type d'Allan (ADEV) de ce type de dispositif, mesurés à l'aide d'un analyseur de bruit (modèle Symmetricom 5125A), pour un décalage de fréquence de $2,1 \times 10^{-12}$. Les courbes montrent que le bruit de l'instrument est inférieur de plus d'un ordre de grandeur à celui d'un maser à hydrogène. Deux modèles de cet instrument ont été construits, afin de produire deux échelles de temps

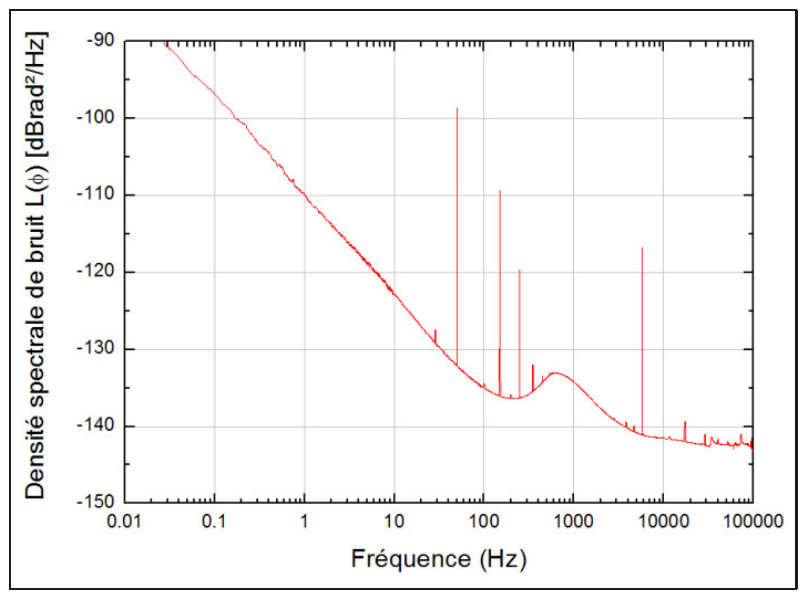

Fig. 3. - Densité spectrale de bruit de phase du plancher de bruit du générateur d'écart de fréquence, mesuré à $100 \mathrm{MHz}$, à l'aide d'un analyseur de bruit Symmetricom 5125A, pour un écart de fréquence appliqué de $2,1 \times 10^{-12}$.

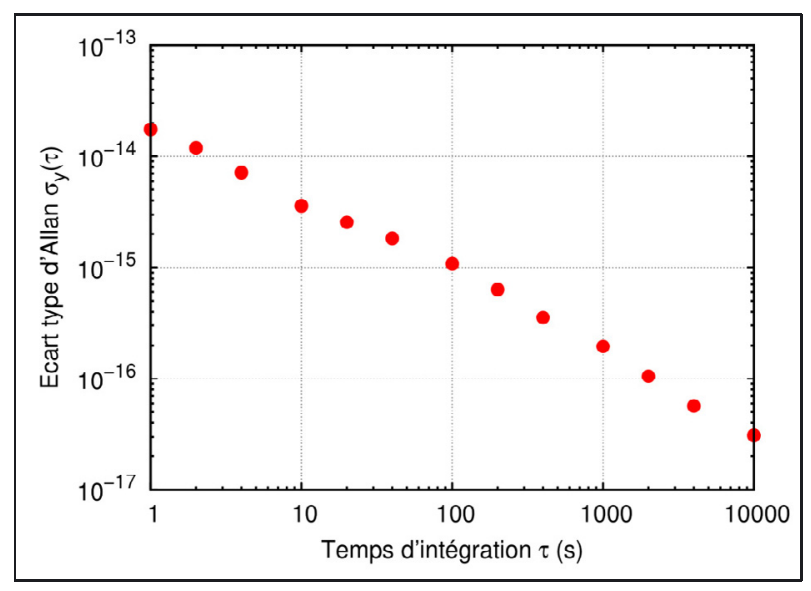

Fig. 4. - Écart type d'Allan du plancher de bruit du générateur d'écarts de fréquence, pour un écart de fréquence appliqué de $2,1 \times 10^{-12}$. Une stabilité meilleure que $10^{-16}$ est atteinte pour des temps d'observation supérieurs à $2000 \mathrm{~s}$.

sur la base des signaux à $100 \mathrm{MHz}$ fournis par deux masers distincts.

En collaboration avec la même société, nous avons aussi développé un commutateur permettant de basculer de l'une à l'autre des deux échelles de temps, avec un impact négligeable sur la phase et l'amplitude de l'ensemble des signaux de UTC(OP). Dans la version antérieure de UTC(OP), comme dans la plupart des laboratoires, un système de secours est maintenu en fonctionnement parallèle, afin de pouvoir le connecter à la distribution des signaux en cas de défaillance de la chaîne de génération principale. La faiblesse de cette approche traditionnelle provient du fait qu'un changement de chaîne entraîne une interruption du signal, au moins sur une courte période, que les délais de distribution sont susceptibles de changer et doivent donc être mesurés à nouveau, et que le niveau des signaux ne sera pas forcément strictement identique. Ce type d'opération de basculement doit donc en général être planifié bien à l'avance, en concertation avec les multiples utilisateurs du signal. 
Le nouveau dispositif de commutation que nous avons développé apporte plus de souplesse par rapport à cette éventualité. L'instrument dispose de deux entrées à $100 \mathrm{MHz}$, que l'on alimente avec les deux échelles de temps générées en parallèle. Il inclut un oscillateur pouvant être verrouillé en phase sur l'une ou l'autre de ces entrées et fournit en sortie trois types de signaux cohérents $(100 \mathrm{MHz}, 10 \mathrm{MHz}$ et $1 \mathrm{PPS})$ alimentant la distribution de UTC(OP). Le commutateur effectue de plus des mesures internes des écarts de phase entre les deux entrées à $100 \mathrm{MHz}$ et le signal généré, avec une résolution de l'ordre de la picoseconde. Cette fonction est utilisée pour effectuer un calage à quelques picosecondes près entre les échelles de temps nominale et redondante avant de commuter. Ainsi, une commutation aura un impact négligeable sur la phase du signal de sortie et le niveau sera identique par construction. Un exemple de commutation est décrit en détail au paragraphe 4.3.

La mise en place d'un logiciel de basculement automatique en cas de détection d'une défaillance serait envisageable. Nous devons cependant acquérir plus d'expérience sur le système actuel. Nous estimons qu'une surveillance attentive des paramètres des masers et des comparaisons de phase nous permet d'anticiper des maintenances préventives. Par ailleurs, l'apparition d'une défaillance soudaine est relativement rare et les causes et conséquences sont en général différentes. Nous préférons donc prendre ce risque, plutôt que de développer un système trop compliqué qui pourrait avoir un comportement incontrôlé si des cas d'erreur non anticipés apparaissaient.

\subsection{Nouvelle chaîne de distribution de UTC(OP)}

Grâce aux nouveaux instruments mis en place pour générer UTC(OP), l'échelle de temps est matérialisée par trois types de signaux cohérents en phase. Ces signaux sont distribués par des amplificateurs de distribution commerciaux choisis pour atteindre les meilleures performances en terme de plancher de bruit de phase. Nous utilisons des distributeurs HPDA-100RM-A de chez SpectraDynamics pour les signaux $100 \mathrm{MHz}$, des distributeurs RF Timetech pour les signaux $10 \mathrm{MHz}$, et des distributeurs de signaux 1 PPS 16 voies de chez Timetech, qui présentent des temps de montée de 500 ps. Nous avons aussi profité de la mise en place de la nouvelle distribution des signaux pour mieux définir le point de référence de UTC $(\mathrm{OP})$. Dans l'ancienne distribution, il était défini à l'entrée du compteur d'intervalles de temps principal servant à effectuer les comparaisons horaires des horloges du laboratoire. Dans le nouveau système, il est matérialisé sur le connecteur de sortie « 16 » du distributeur de signaux 1 PPS, à un niveau de déclenchement de $1 \mathrm{~V}$ lorsque le signal est chargé sur $50 \Omega$. Cette sortie de référence est laissée en permanence disponible afin de pouvoir effectuer des mesures. Ainsi, avec cette nouvelle définition, l'exactitude sur la mesure d'un délai par rapport à ce point de référence est limitée par l'équipement utilisé et ne dépend plus du comportement de la voie d'entrée du compteur d'intervalles de temps. Si nécessaire, nous pouvons effectuer des étalonnages par rapport

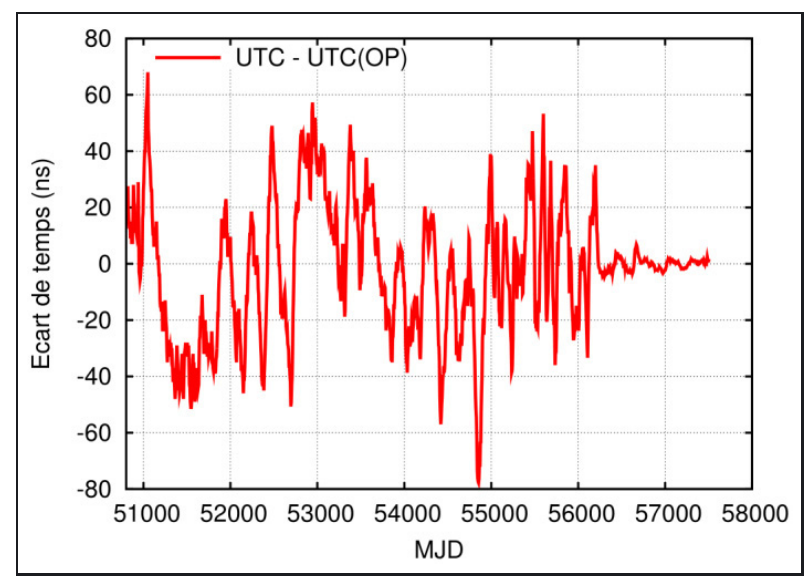

Fig. 5. - Écart de temps entre UTC(OP) et l'UTC depuis 1998. La nouvelle méthode de génération de UTC $(\mathrm{OP})$ a été mise en place à partir du MJD 56229.

au point de référence de $\mathrm{UTC}(\mathrm{OP})$ en utilisant un dateur d'évènements haute résolution [14] et en appliquant une méthode de mesure différentielle.

Depuis la première mise en place du nouvel UTC(OP) en octobre 2012, il n'y a eu qu'un changement mineur dans l'algorithme. Il s'agissait, comme indiqué au paragraphe 2, de prendre en compte le fait que les premiers points publiés dans la Circulaire $\mathrm{T}$, sont obtenus avec un pilotage différent de celui de la Circulaire T précédente. Lors des premiers mois de fonctionnement, le logiciel réalisant le FLL et le PLL pour la mise à jour mensuelle du pilotage de UTC(OP) vers l'UTC, consistait à lancer différents sous-programmes. Dorénavant, après plusieurs améliorations successives, ces tâches ont été regroupées dans un script unique qu'il suffit de lancer dès que la Circulaire $\mathrm{T}$ est disponible sur le site internet du BIPM. Rappelons aussi que les traitements de données fournissent quotidiennement l'étalonnage de fréquence de chaque maser par rapport à toutes les fontaines atomiques. En pratique, le système actuel produit les corrections de fréquence pour générer quatre échelles de temps basées sur nos quatre masers : les deux échelles de temps nominale et redondante utilisant les générateurs d'écart de fréquence $100 \mathrm{MHz}$, et deux autres exploitant les synthétiseurs $5 \mathrm{MHz}$ ancienne génération, utilisées pour des expérimentations et pour de la redondance supplémentaire. Ainsi, quand cela est nécessaire, il est très facile de changer la fontaine de référence ou le maser à hydrogène de l'une des échelles de temps, en modifiant un fichier de paramètres.

\section{Les performances de $\mathrm{UTC}(\mathrm{OP})$}

\subsection{Caractérisation dans le domaine temporel}

Comme indiqué dans le paragraphe 2, l'objectif principal de UTC(OP) est de fournir une réalisation en temps réel de UTC avec la meilleure approximation possible. L'indicateur le plus significatif de cette caractéristique peut être tout simplement l'écart de temps entre UTC(OP) et l'UTC. La figure 5 présente 


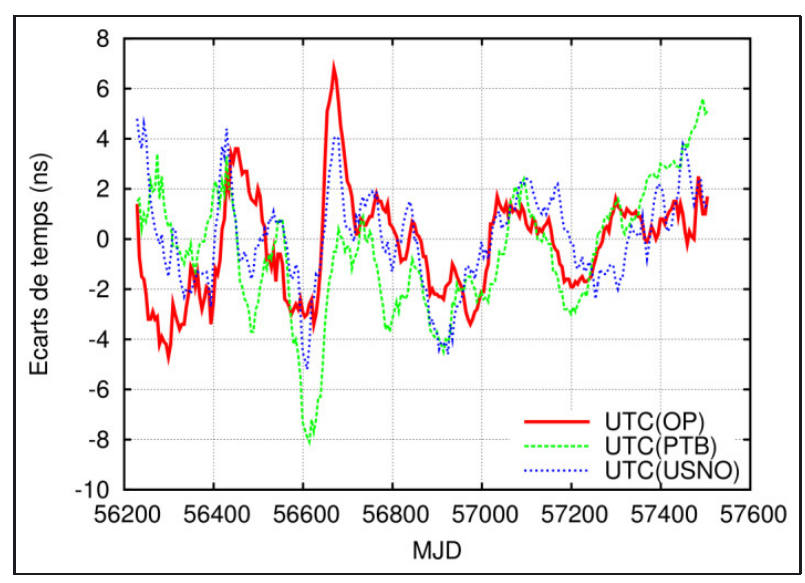

Fig. 6. - Comparaison de temps à l'UTC de trois $\operatorname{UTC}(k)$ fondés sur l'exploitation de fontaines atomiques, depuis la mise en place du nouvel algorithme pour la génération de UTC(OP).

une comparaison à long terme depuis 1998 de UTC(OP) à l'UTC. La courbe montre une amélioration de plus d'un ordre de grandeur du nouvel UTC(OP), mis en place à partir du MJD 56229, par rapport au système précédent basé sur une horloge commerciale à jet thermique de césium. La figure 6 présente cette comparaison, depuis la mise en place du nouvel UTC(OP) en octobre 2012, ainsi que les performances de deux autres $\mathrm{UTC}(k)$ fondés également sur l'exploitation de fontaines atomiques [15-17]. Sur les trois dernières années, UTC(OP) s'est maintenu à mieux que $10 \mathrm{~ns}$ de l'UTC, avec une valeur moyenne de $0,33 \mathrm{~ns}$, et un écart type de 2,1 ns. La différence est même inférieure à $5 \mathrm{~ns}$, si l'on exclut une courte période autour du MJD 56650. On observe que les autres $\operatorname{UTC}(k)$, qui présentent des performances similaires à celles de UTC(OP), comportent une variation comparable autour de cette même période. Au cours de l'année 2015, l'écart entre UTC $(\mathrm{OP})$ et l'UTC est même resté inférieur à 2 ns. Ce niveau de performance s'approche de l'incertitude des moyens de transfert de temps.

\subsection{Caractérisation dans le domaine fréquentiel}

La figure 7 présente l'évolution de la stabilité relative de fréquence de $[U T C-U T C(O P)]$, en se basant sur les données de la Circulaire T. Les carrés représentent l'écart type d'Allan calculé sur l'ensemble de la période postérieure à la mise en place du nouvel algorithme. Les triangles portent sur la période d'après janvier 2014 (MJD 56659), date à laquelle un nouvel algorithme de pondération des horloges a été mis en place dans le calcul de l'UTC par le BIPM [18]. Les ronds correspondent à l'année 2015. Ces courbes montrent une stabilité à 5 jours inférieure ou égale à $10^{-15}$. Elle atteint $2-5 \times 10^{-16}$ pour des périodes d'observation supérieure à 100 jours, grâce au pilotage vers l'UTC. Bien qu'aucun évènement marquant n'ait été identifié pour expliquer l'amélioration des résultats au cours de l'année 2015, nous pouvons supposer que ceci provient de l'amélioration progressive des instruments utilisés pour générer et distribuer UTC(OP),

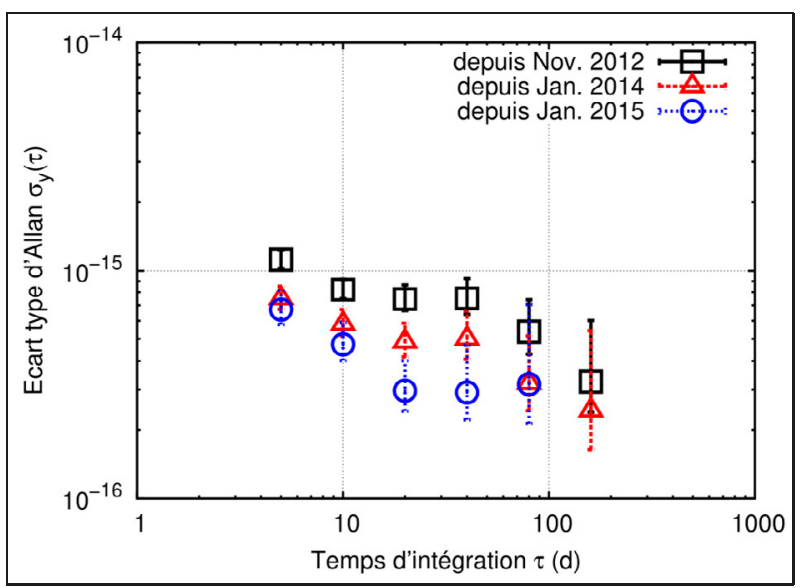

Fig. 7. - Stabilité de fréquence relative de $[U T C-U T C(O P)]$ : la courbe noire porte sur l'ensemble des données depuis novembre 2012 ; la courbe rouge a été calculée en prenant les données depuis janvier 2014 ; la courbe bleue représente la stabilité au cours de l'année 2015.

qui présentent en particulier de plus faibles sensibilités en température.

La figure 8 présente la caractérisation des nouveaux dispositifs mis en place, effectuée en analysant les résultats de mesures obtenus sur la période MJD 5737557404. Les lignes correspondent aux ADEV des comparaisons à UTC(OP) des deux entrées du commutateur alimentées par les générateurs d'écart de fréquence SKK1 et SKK2, mesurées grâce à des comparateurs de phase de signaux à $100 \mathrm{MHz}$ (PCO Timetech). Les cercles correspondent à l'analyse des mesures horaires sur les signaux 1 PPS avec un compteur d'intervalles de temps. Pour l'analyse des mesures PCO, étant donné la quantité de données (une mesure par seconde), nous avons tracé deux séries de courbes dans chaque cas. Pour le long terme, nous avons calculé l'écart type d'Allan des données après avoir effectué des moyennes sur 0,01 jour. Pour le court terme, les écarts types d'Allan présentés correspondent aux données obtenues au cours d'une journée incluse dans la période.

Sur la période considérée, $\mathrm{UTC}(\mathrm{OP})$ reposait sur SKK2 alimenté par le maser 810. Les courbes verte et rouge présentent la stabilité de la comparaison de SKK1, alimenté par le maser 889 , à UTC(OP). Sur le court terme, elles correspondent donc au bruit de la comparaison entre deux masers à hydrogène, variant de $10^{-13}$ à une seconde jusqu'à $10^{-15}$ à un jour. Sur des périodes d'observation plus longues, la stabilité descend jusque $10^{-16}$, grâce au pilotage en fréquence journalier utilisant les données de fontaines atomiques, qui permet de compenser les dérives de fréquence des deux masers à hydrogène. Pour les courbes relatives à [SKK2 $U T C(O P)]$, le bruit du maser ainsi que celui du générateur d'écart de fréquence sont rejetés, car ils sont en mode commun. La stabilité part de $2 \times 10^{-14}$ à une seconde et descend dans la gamme des $10^{-18}$, pour des périodes supérieures à quelques jours. Ces résultats sont à la limite du plancher de bruit d'un PCO. Les cercles 


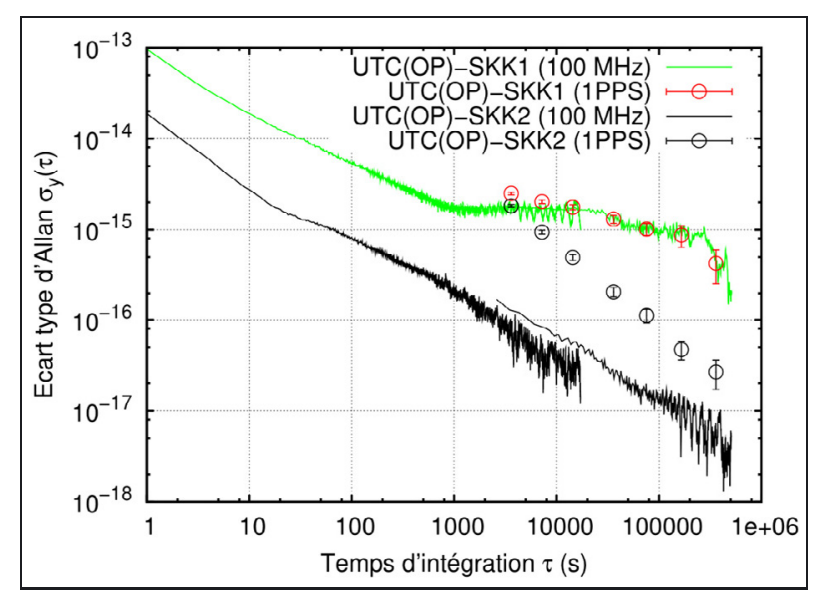

Fig. 8. - Stabilité de fréquence relative de UTC(OP) par rapport à l'échelle de temps de redondance SKK1 (vert et rouge) ; bruit résiduel de la chaîne de génération et de distribution, mesuré par rapport à l'échelle de temps SKK2 utilisée pour produire UTC(OP); les comparaisons à $100 \mathrm{MHz}$ ont été effectuées à l'aide d'un PCO et les mesures 1 PPS avec un compteur d'intervalles de temps SR620.

noirs correspondent au bruit du compteur d'intervalle de temps, qui atteint $10^{-16}$ après un temps d'intégration de un jour. Le bruit des générateurs d'écart de fréquence a été caractérisé auparavant (fig. 3 et 4). Ces résultats démontrent que l'ensemble des nouveaux instruments développés présente un plancher de bruit inférieur d'un ordre de grandeur à celui d'un maser sur toutes les périodes d'observation. De plus, les masers à hydrogène et les comparateurs de phase PCO sont situés dans une salle en sous-sol du bâtiment, alors que le système de génération et de distribution des signaux de UTC $(\mathrm{OP})$ sont localisés deux étages au-dessus. Les résultats nous fournissent donc une validation de l'installation des câbles à mieux que $10^{-17}$. L'une des motivations du développement des nouveaux instruments et de la distribution des signaux était aussi de se préparer à la mission spatiale «Atomic Clock Ensemble in Space» (ACES) [19]. Les résultats obtenus sont tout à fait compatibles avec les spécifications de la station sol du lien micro-onde de cette mission. Grâce aux améliorations apportées, nous pourrons fournir UTC(OP) comme référence à la station qui sera installée en 2017 sur le toit d'un autre bâtiment de l'Observatoire de Paris. Nous prévoyons de transmettre la référence à $100 \mathrm{MHz}$ au moyen d'un lien fibré stabilisé modulé à $1 \mathrm{GHz}$.

\subsection{Basculement du commutateur}

La figure 9 présente les performances du nouveau système de génération de UTC(OP) dans le cas réel d'un basculement entre les échelles de temps nominale (maser 889 piloté grâce à SKK1) et redondante (maser 810 alimentant SKK2). En effet, à cause de la défaillance d'une carte électronique du maser 889 servant à la surveillance de ses paramètres internes, nous avons décidé d'effectuer cette commutation de SKK1 à SKK2 au MJD 57364, afin de pouvoir effectuer en toute sérénité la réparation. Juste avant de commander la commutation, nous avons

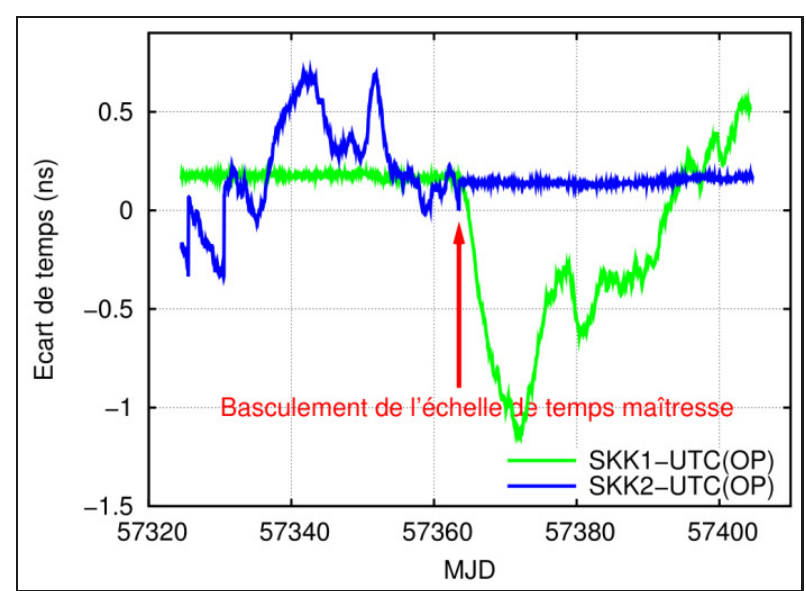

Fig. 9. - Écarts de temps entre UTC(OP) et les deux échelles de temps SKK1 et SKK2 : La source de UTC(OP) était fournie par SKK1 jusqu'au MJD 57364 ; SKK2 constituait alors l'échelle de temps de secours; les rôles ont été intervertis au MJD 57364 en jouant sur le commutateur.

aligné SKK2 sur SKK1 en appliquant un saut de phase de 200 ps, en se reposant sur les mesures internes du commutateur. Après réparation, le maser 889 a été redémarré et l'échelle de temps SKK1 a été alignée à nouveau sur UTC(OP). Nous observons sur la figure 9, que SKK1 présente une excursion de l'ordre de 1 ns après la réparation. Ceci est probablement dû à une petite variation de la fréquence du maser 889, qui a été arrêté pendant environ deux heures durant l'opération. Après quelques jours de pilotage automatique, la différence entre les deux échelles de temps nominale et redondante s'est stabilisée et s'est réduite à moins de $1 \mathrm{~ns}$, ce qui indique que nous pourrions revenir sur l'échelle de temps SKK1 pour réaliser $\mathrm{UTC}(\mathrm{OP})$.

Plus généralement, la figure 9 donne les performances pouvant être atteintes par deux échelles de temps fondées sur deux masers à hydrogène distincts, que l'on peut considérer comme indépendantes sur des périodes supérieures à 10 jours. Bien qu'elles reposent sur les masers les plus prédictibles dont nous disposons, des fluctuations de l'ordre de la nanoseconde sont observées. Celles-ci sont dues principalement au comportement propre des masers à hydrogène.

\section{Conclusion}

Nous avons décrit le nouvel algorithme mis en place pour la génération de UTC(OP). Celui-ci repose sur un feed-forward permettant de compenser chaque jour la dérive de fréquence d'un maser à hydrogène en s'appuyant sur les données d'étalonnage des fontaines atomiques du LNE-SYRTE. Un pilotage mensuel fin, sur la base de la somme d'un FLL et d'un PLL utilisant des résultats de la Circulaire $\mathrm{T}$, permet de maintenir $\mathrm{UTC}(\mathrm{OP})$ très proche de l'UTC.

Le nouvel algorithme a été mis en place en octobre 2012, en utilisant l'instrumentation initiale fonctionnant à $5 \mathrm{MHz}$. Tous les équipements ont ensuite 
été progressivement remplacés par de plus performants, pour certains développés spécialement pour cette application. En particulier, nous avons développé un nouveau type de générateurs d'écart de fréquence fonctionnant à $100 \mathrm{MHz}$, dont le niveau de bruit est négligeable devant celui d'un maser à hydrogène. Le système est complété par un commutateur permettant de basculer entre deux échelles de temps nominale et redondante, avec une continuité de phase de l'ordre de la picoseconde, garantissant la continuité de la distribution du signal. La période de transition est désormais achevée. Le nouvel UTC(OP) constitue aujourd'hui la référence unique distribuée aux moyens de transfert de temps et aux utilisateurs sous la forme de signaux à $100 \mathrm{MHz}, 10 \mathrm{MHz}$ et $1 \mathrm{PPS}$, qui ont été caractérisés aux différents étages du système. La stabilité de UTC(OP) est une réplique de celle du maser à hydrogène sur des périodes d'analyse inférieures à quelques jours. Sur des périodes plus longues, elle est bien meilleure que celle d'un maser libre, car elle rejoint la stabilité à long terme des fontaines atomiques du laboratoire. Les stabilités de fréquence mesurées montrent que le signal à $100 \mathrm{MHz}$ de UTC(OP) est compatible avec les spécifications du terminal sol du lien micro-onde de la mission spatiale ACES.

Sur ses trois premières années de fonctionnement, le nouvel UTC(OP) a été l'une des meilleures réalisations temps réel de l'UTC, s'en écartant de moins de 10 ns, grâce au fonctionnement quasi continu des fontaines atomiques du LNE-SYRTE. Au cours de l'année 2015, cet écart s'est même maintenu à moins de 2 ns. Ces performances atteignent dorénavant l'incertitude des moyens de transfert de temps opérationnels qui deviennent le facteur limitant dans l'amélioration des performances des $\operatorname{UTC}(k)$.

\section{Avertissement}

Les noms et les modèles des équipements cités dans cet article sont donnés uniquement à titre indicatif, sans aucun caractère de critique ou de publicité.

\section{Remerciements}

Les auteurs tiennent à remercier l'appui du Service d'électronique du LNE-SYRTE dirigé par Michel Lours, ainsi que le personnel technique du service des Références nationales de temps. Nous remercions également Marco Siccardi pour de fructueuses discussions, ainsi que pour le développement des générateurs d'écart de fréquence et du commutateur par sa société. Daniele Rovera remercie Iakira Couceiro de l'institut brésilien INMETRO pour l'opportunité de mesurer le laser He-Ne stabilisé sur une raie de l'iode.

\section{Références}

[1] QUINN T.J., "News from the BIPM", Metrologia, 31, 55, 1994, DOI:10.1088/0026-1394/31/1/012.

[2] Uhrich P., Valat D., Brunet M., Marechal J., BELTAN T. et SuARD N., "The French time reference UTC(OP), and the connection of the EGNOS Network Time", $19^{\text {th }}$ European Frequency and Time Forum (EFTF), 2005, 643-648.
[3] PARKer T. et LeVINE J., "Impact of new high stability frequency standards on the performance of the NIST AT1 time scale", IEEE Transactions on Ultrasonics, Ferroelectrics, and Frequency Control, 44, 1997, 12391244, ISSN 0885-3010, DOI:10.1109/58.656627.

[4] Clairon A., Ghezali S., Santarelli G., Laurent P., Lea S., Bahoura M., Simon E., Weyers S. et SZYMANIEC K., "Preliminary accuracy evaluation of a cesium fountain frequency standard", Proc. of $5^{\text {th }}$ Symposium on Frequency Standards and Metrology, Ed. Bergquist J. (Singapore: World Scientific), 1996.

[5] Bize S., Sortais Y., Santos M., Mandache C., Clairon A. et SALOMON C., "High-accuracy measurement of the ${ }^{87} \mathrm{Rb}$ ground-state hyperfine splitting in an atomic fountain", Europhys. Lett., 45, 558, 1999, DOI:10.1209/epl/11999-00203-9.

[6] Guéna J., Rosenbusch P., Laurent P., AbGrall M., Rovera D., Santarelli G., Tobar M., Bize S. et ClaIron A., "Demonstration of a dual alkali $\mathrm{Rb} / \mathrm{Cs}$ fountain clock", IEEE Transactions on Ultrasonics, Ferroelectrics and Frequency Control, 57, 2010, 647-653, ISSN 0885-3010, DOI:10.1109/TUFFC.2010.1461.

[7] Guéna J., Abgrall M., Rovera D., Laurent Ph., Chupin B., Lours M., SANTARelli G., Rosenbusch P., Tobar M., Li R., Gibble K., Clairon A. et BIZE S., "Progress in atomic fountains at LNE-SYRTE", IEEE Transactions on Ultrasonics, Ferroelectrics, and Frequency Control, 59, 2012, 391-409, ISSN 0885-3010, DOI:10.1109/TUFFC.2012.2208.

[8] Guéna J., Abgrall M., Clairon A. et Bize S., "Contributing to TAI with a secondary representation of the SI second", Metrologia, 51, 108, 2014, DOI:10.1088/0026-1394/51/1/108.

[9] Rovera D., "Aplicaçéões metrológicas de um pente de frequências", Tech. Rep., CNPq:551404/2011-6 INMETRO, 2012, in Portuguese.

[10] Franklin G.F., Powell J.D. et Emami-Naeini A., "Feedback control of dynamic systems", Addison-Welsey, 1986.

[11] MANN A.G., "Ultrastable cryogenic microwave oscillators", Frequency measurement and control, collection Topics Appl. Phys., 79, ed. Luiten A. (Springer-Verlag), 2000, 37-67.

[12] Santarelli G., Laurent Ph., Lemonde P., Clairon A., ManN A.G., Chang S., LUiten A.N. et SAlomon C., "Quantum Projection Noise in an Atomic Fountain: A High Stability Cesium Frequency Standard", Phys. Rev. Lett., 82, 1999, 4619, DOI:10.1103/PhysRevLett.82.4619.

[13] Rovera G., AbGRall M. et Siccardi M., "Characterization of an auxiliary offset generator for steering of $\mathrm{H}$ Masers", $26^{\text {th }}$ European Frequency and Time Forum (EFTF), 2012, 14-15.

[14] SAMain E., Fridelance P. et Guillemot Ph., "An ultrastable event timer designed for T2L2", $24^{\text {th }}$ European Frequency and Time Forum (EFTF), 2010, 1-8. 
[15] Bauch A., Weyers S., Piester D., Staliuniene E. et YANG W., "Generation of UTC(PTB) as a fountainclock based time scale", Metrologia, 49, 180, 2012, DOI:10.1088/0026-1394/49/3/180.

[16] Peil S., Hanssen J.L., Swanson T.B., TaYlor J. et EKSTROM C.R., "Evaluation of long term performance of continuously running atomic fountains", Metrologia, 51, 263, 2014, DOI:10.1088/0026-1394/51/3/263.

Article reçu le 13 juin 2016, version révisée reçue le 4 novembre 2016.
[17] Rovera G.D., Bize S., Chupin B., GuÉna J., LAURENT Ph., Rosenbusch P., Uhrich P. et ABgRall M., "UTC(OP) based on LNE-SYRTE atomic fountain primary frequency standards", Metrologia, 53, S81, 2016, DOI:10.1088/0026-1394/53/3/S81.

[18] Panfilo G., Harmegnies A. et Tisserand L., "A new weighting procedure for UTC", Metrologia, 51, 285, 2014, DOI:10.1088/0026-1394/51/3/285.

[19] Salomon C., Cacciapuoti L. et DimarQ N., "Atomic Clock Ensemble in Space: an Update", Int. J. Mod. Phys. D, 16, 2007, 2511-2523, DOI:10.1142/S0218271807011681. 